\title{
Complete genome sequence of the sulfate-reducing firmicute Desulfotomaculum ruminis type strain $\left(\mathrm{DL}^{\mathrm{T}}\right)$
}

\author{
Stefan Spring ${ }^{1}$, Michael Visser ${ }^{2}$, Megan Lu $^{3,4}$, Alex Copeland ${ }^{3}$, Alla Lapidus ${ }^{3}$, Susan Lucas ${ }^{3}$, \\ Jan-Fang Cheng ${ }^{3}$, Cliff Han $^{3,4}$, Roxanne Tapia ${ }^{3,4}$, Lynne A. Goodwin ${ }^{3,4}$, Sam Pitluck ${ }^{3}$, Natalia \\ Ivanova $^{3}$, Miriam Land ${ }^{3,5}$, Loren Hauser ${ }^{3,5}$, Frank Larimer ${ }^{3,5}$, Manfred Rohde ${ }^{6}$, Markus \\ Göker ${ }^{1}$, John C. Detter ${ }^{3,4}$, Nikos C. Kyrpides ${ }^{3}$, Tanja Woyke ${ }^{3}$, Peter J. Schaap ${ }^{7}$, Caroline M. \\ Plugge $^{2}$, Gerard Muyzer ${ }^{8}$, Jan Kuever ${ }^{9}$, Inês A. C. Pereira ${ }^{10}$, Sofiya N. Parshina ${ }^{11}$, Rizlan \\ Bernier-Latmani $^{12}$, Alfons J.M. Stams ${ }^{2 *}$, Hans-Peter Klenk ${ }^{1 *}$ \\ ${ }^{1}$ Leibniz Institute DSMZ - German Collection of Microorganisms and Cell Cultures, \\ Braunschweig, Germany \\ ${ }^{2}$ Laboratory of Microbiology, Wageningen University, Wageningen, The Netherlands \\ ${ }^{3}$ DOE Joint Genome Institute, Walnut Creek, California, USA \\ ${ }^{4}$ Los Alamos National Laboratory, Bioscience Division, Los Alamos, New Mexico, USA \\ ${ }^{5}$ Oak Ridge National Laboratory, Oak Ridge, Tennessee, USA \\ ${ }^{6}$ HZI - Helmholtz Centre for Infection Research, Braunschweig, Germany \\ ${ }^{7}$ Laboratory of Systems and Synthetic Biology, Wageningen University, Wageningen, The \\ Netherlands \\ ${ }^{8}$ Department of Aquatic Microbiology, Institute for Biodiversity and Ecosystem Dynamics, \\ University of Amsterdam, Amsterdam, The Netherlands \\ ${ }^{9}$ Department of Microbiology, Bremen Institute for Materials Testing, Bremen, Germany \\ ${ }^{10}$ Instituto de Tecnologia Quimica e Biologica, Universidade Nova de Lisboa, Oeiras, \\ Portugal \\ ${ }^{11}$ Wingradsky Institute of Microbiology Russian Academy of Sciences, Moscow, Russia \\ ${ }^{12}$ Ecole Polytechnique Federale de Lausanne, Lausanne, Switzerland
}

*Corresponding authors: Alfons J.M. Stams and Hans-Peter Klenk

Keywords: anaerobic, motile, sporulating, mesophilic, sulfate-reducer, hydrogen sulfide, incomplete oxidizer, mixotrophic, CSP 2009, Peptococcaceae, Clostridiales

Desulfotomaculum ruminis Campbell and Postgate 1965 is a member of the large genus Desulfotomaculum which contains 30 species and is contained in the family Peptococcaceae. This species is of interest because it represents one of the few sulfatereducing bacteria that have been isolated from the rumen. Here we describe the features of $D$. ruminis together with the complete genome sequence and annotation. The 3,969,014 bp long chromosome with a total of 3,901 protein-coding and 85 RNA genes is the second completed genome sequence of a type strain of the genus Desulfotomaculum to be published, and was sequenced as part of the DOE Joint Genome Institute Community Sequencing Program 2009.

\section{Introduction}

Strain DL D $^{\mathrm{T}}$ = DSM $2154=$ ATCC $23193=$ NCIMB 8452 ) is the type strain of the species Desulfotomaculum ruminis [1], one out of currently 30 species with validly published names in the paraphyletic genus Desulfotomaculum [2,3]. Strain $\mathrm{DL}^{\mathrm{T}}$ was initially isolated by G. S. Coleman in the 1950s from the rumen of hay-fed sheep [4]. Dissimilatory reduction of sulfate to sulfide in the rumen was first demonstrated by Lewis [5], who dosed fistulated sheep with sulfate and determined the amount of sulfide produced. As high amounts of sulfide may be toxic to animals, bacterial sulfate-reduction in ruminants was a concern due to the presence of sulfate in grass and hay. $D$. ruminis represented the first pure culture of a sulfate-reducing bacterium isolated from the rumen. The genus name was derived from the Latin words 'de', from, 'sulfur', sulfur, and 'tomaculum', 
a kind of sausage, meaning 'a sausage-shaped sulfate reducer' $[2,6]$. The species epithet is derived from the Latin word 'rumen', throat, first stomach (rumen) of a ruminant, meaning of a rumen [1,2]. Here, we present a summary classification and a set of features for D. ruminis strain $\mathrm{DL}^{\mathrm{T}}$, together with the description of the complete genomic sequencing and annotation. The complete genome sequence of strain $\mathrm{DL}^{\mathrm{T}}$ will provide valuable information for defining a more adequate description of the currently paraphyletic genus Desulfotomaculum.

\section{Classification and features}

A representative genomic 16S rRNA sequence of D. ruminis DSM $2154^{\mathrm{T}}$ was compared using NCBI BLAST $[7,8]$ under default settings (e.g., considering only the high-scoring segment pairs (HSPs) from the best 250 hits) with the most recent release of the Greengenes database [9] and the relative frequencies of taxa and keywords (reduced to their stem [10]) were determined, weighted by BLAST scores. The most frequently occurring genera were Desulfotomaculum (88.3\%), Pelotomaculum (7.9\%), Cryptanaerobacter (2.8\%) and 'Catabacter' (1.0\%) (60 hits in total). Regarding the four hits to sequences from members of the species, the average identity within HSPs was $99.1 \%$, whereas the average coverage by HSPs was $86.1 \%$. Regarding the 41 hits to sequences from other members of the genus, the average identity within HSPs was $93.2 \%$, whereas the average coverage by HSPs was $90.7 \%$. Among all other species, the one yielding the highest score was Desulfotomaculum putei (HM228397), which corresponded to an identity of $94.1 \%$ and an HSP coverage of $98.5 \%$. (Note that the Greengenes database uses the INSDC (= EMBL/NCBI/DDBJ) annotation, which is not an authoritative source for nomenclature or classification.) The highestscoring environmental sequence was EU307084 ('Changes microbial metabolic and along hydrogeochemically variable profile unsaturated horizon soil aggregate clone A Ac-2 12'), which showed an identity of $97.5 \%$ and an HSP coverage of 98.4. D. ruminis has not only been found in the rumen of animals, but also in other environments $[11,12]$. Therefore, the distribution of $D$. ruminis is not restricted to the rumen of animals. Hence, it is likely that this species enters the digestive tract of ruminants via food contaminated by spores.

Figure 1 shows the phylogenetic neighborhood of D. ruminis in a $16 \mathrm{~S}$ rRNA based tree of type strains. The sequences of the five $16 \mathrm{~S}$ rRNA gene copies in the genome differ from each other by up to two nucleotides, and differ by up to three nucleotides from the previously published 16S rRNA sequence (Y11572), which contains three ambiguous base calls.

Cells of $D$. ruminis $\mathrm{DL}^{\mathrm{T}}$ are slightly curved rods with rounded ends 2-6 $\mu \mathrm{m}$ in length and 0.5-0.7 $\mu \mathrm{m}$ in width (Table 1 and Figure 2) [1,4]. Cells stain Gram-negative and form oval subterminal spores that slightly swell the cells. A slight tumbling motility is due to peritrichous flagellation [1]. Strain $\mathrm{DL}^{\mathrm{T}}$ grows optimally at $37^{\circ} \mathrm{C}$, but not above $48^{\circ} \mathrm{C}$ [1]. The $\mathrm{pH}$ range for growth is $6.0-8.5$ with an optimum between $\mathrm{pH} 6.0$ and 7.0 [4]. $D$. ruminis strains are obligately anaerobic and can grow chemoheterotrophically with lactate, pyruvate, ethanol or alanine as well as mixotrophically with hydrogen or formate as electron donor and acetate as carbon source. In contrast to the distantly related $D$. acetoxidans, strains of $D$. ruminis oxidize substrates incompletely to acetate and cannot grow autotrophically [4]. Suitable electron acceptors are sulfate, thiosulfate and sulfite, but not elemental sulfur or nitrate $[1,26]$. Fermentative growth with pyruvate as sole substrate is also possible [26].

\section{Chemotaxonomy}

In cells of $D$. ruminis cytochromes of the $b$-type dominate [1], which is a typical trait of sulfatereducing bacteria belonging to the genus Desulfotomaculum. Respiratory lipoquinones are also present and are comprised mainly of the menaquinone MK-7 and some small amounts of MK-6 [27]. The whole-cell fatty acid pattern of the type strain of $D$. ruminis was determined by Hagenauer et al. [26], who found a dominance of branched-chain iso- and anteiso-fatty acids in addition to unsaturated fatty acids, whereas saturated unbranched fatty acids were of less importance. The predominant fatty acids were: iso$\mathrm{C}_{17: 1 \mathrm{c} 7}$, iso- $\mathrm{C}_{15: 0}$, iso- $\mathrm{C}_{17: 0}, \mathrm{C}_{17: 0 \text { cyc }}$ and $\mathrm{C}_{16: 0}$. Although, in the study of Hagenauer et al. [26] a large amount of the extracted cellular fatty acids $(37.3 \%)$ remained unidentified, the fatty acid pattern of $D$. ruminis can be clearly distinguished from other distantly related Desulfotomaculum species like $D$. acetoxidans, which has a pattern dominated by straight-chain saturated fatty acids, thus further illustrating the paraphyletic origin of this genus. 


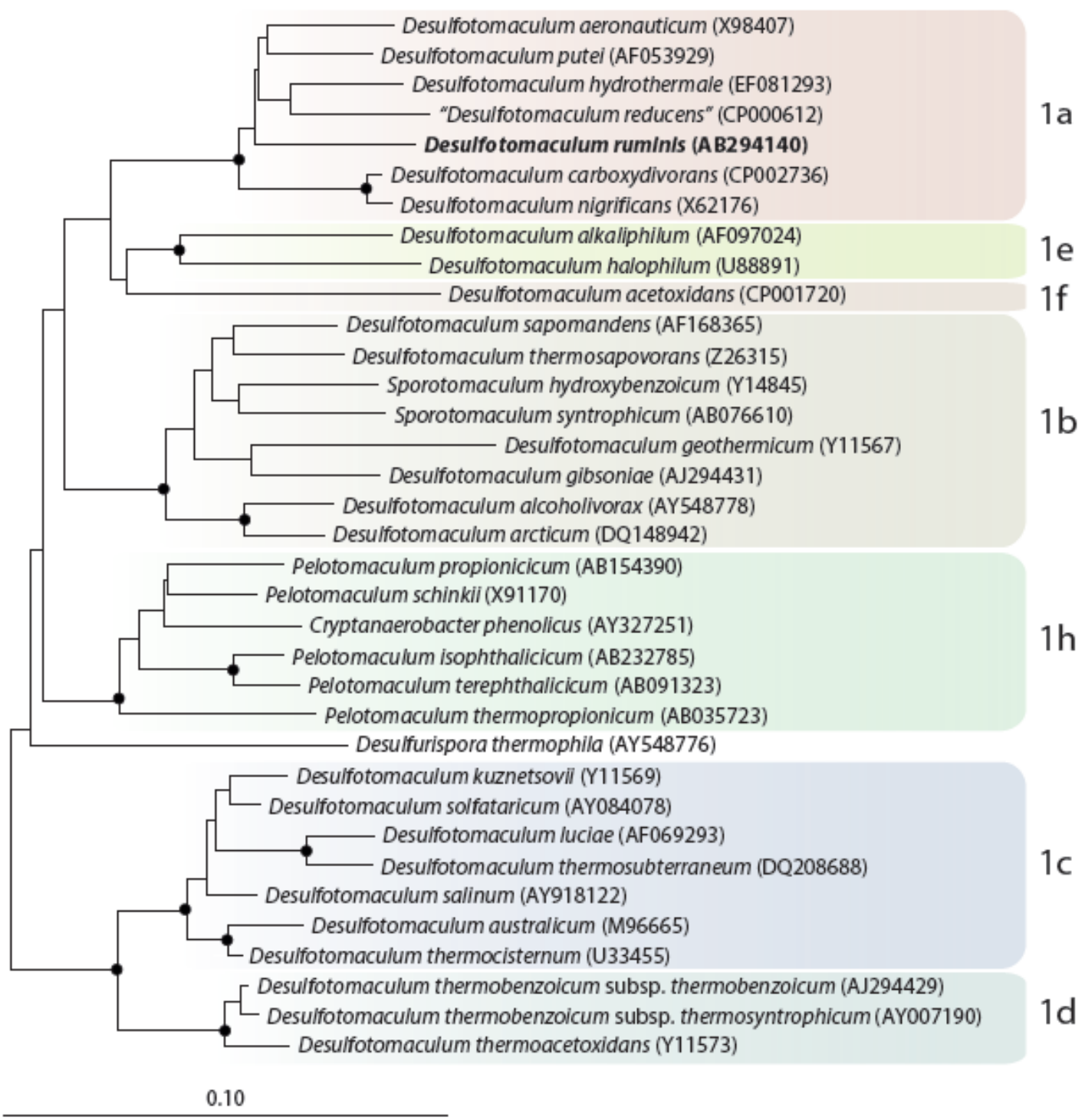

Figure 1. Neighbor-joining tree based on $16 \mathrm{~S}$ rRNA sequences showing the phylogenetic affiliation of Desulfotomaculum and related species. D. ruminis is printed in bold type. The sequences of different Thermotogales were used as outgroup, but were pruned from the tree. Closed circles represent bootstrap values between 75 and $100 \%$. The scale bar represents $2 \%$ sequences difference.

\section{Genome sequencing and annotation}

\section{Genome project history}

This organism was selected for sequencing on the basis of the DOE Joint Genome Institute Community Sequencing Program 2009 proposal 300132_795700 'Exploring the genetic and physiological diversity of Desulfotomaculum species'. The genome project is deposited in the Genomes OnLine Database (Gc01775) [28] and the complete genome sequence is deposited in GenBank (CP002780). Sequencing, finishing and annotation were performed by the DOE Joint Genome Institute (JGI). A summary of the project information is shown in Table 2.

\section{Growth conditions and DNA isolation}

D. ruminis strain DL ${ }^{\mathrm{T}}$, DSM 2154, was grown anaerobically in DSMZ medium 63 (Desulfovibrio medium) [29] at $37^{\circ} \mathrm{C}$. DNA was isolated from 0.5$1.0 \mathrm{~g}$ of cell paste using Jetflex Genomic DNA Purification Kit (GENOMED 600100) following the manufacturer's instructions, with a modified protocol for cell lysis (modification st/LALMP) as described in Wu et al. 2009 [30]. DNA is available through the DNA Bank Network [31]. 
Table 1. Classification and general features of $D$. ruminis $\mathrm{DL}^{\top}$ according to the MIGS recommendations [13] and the NamesforLife database [3].

\begin{tabular}{|c|c|c|c|}
\hline MIGS ID & Property & Term & Evidence code \\
\hline & \multirow{8}{*}{ Current classification } & Domain Bacteria & TAS [14] \\
\hline & & Phylum Firmicutes & TAS [15-17] \\
\hline & & Class Clostridia & TAS $[18,19]$ \\
\hline & & Order Clostridiales & TAS $[20,21]$ \\
\hline & & Family Peptococcaceae & TAS $[20,22]$ \\
\hline & & Genus Desulfotomaculum & TAS $[1,6,20]$ \\
\hline & & Species Desulfotomaculum ruminis & TAS $[1,20]$ \\
\hline & & Type strain DL & TAS [1] \\
\hline & Gram stain & negative & TAS [1] \\
\hline & Cell shape & rod-shaped & TAS [1] \\
\hline & Motility & motile & TAS [1] \\
\hline & Sporulation & sporulating & TAS [1] \\
\hline & Temperature range & $48^{\circ} \mathrm{C}$ is the upper limit & TAS [1] \\
\hline & Optimum temperature & $37^{\circ} \mathrm{C}$ & TAS [1] \\
\hline & Salinity & not reported & \\
\hline \multirow[t]{3}{*}{ MIGS-22 } & Oxygen requirement & obligate anaerobe & TAS [1] \\
\hline & Carbon source & $\begin{array}{l}\text { acetate in combination with } \mathrm{CO}_{2} \text { and a } \\
\text { variety of other organic compounds }\end{array}$ & TAS [23] \\
\hline & Energy metabolism & mixotrophic, heterotrophic & TAS $[1,23]$ \\
\hline MIGS-6 & Habitat & $\begin{array}{l}\text { rumen contents of sheep, fresh water, } \\
\text { mud, sea water, soil }\end{array}$ & TAS [1] \\
\hline MIGS-15 & Biotic relationship & free-living & TAS [1] \\
\hline \multirow[t]{3}{*}{ MIGS-14 } & Pathogenicity & none & TAS [1] \\
\hline & Biosafety level & 1 & TAS [24] \\
\hline & Isolation & rumen of hay-fed sheep & TAS [1] \\
\hline MIGS-4 & Geographic location & Babraham, Cambridgeshire, UK & TAS [4] \\
\hline MIGS-5 & Sample collection time & 1955 or before & TAS [4] \\
\hline MIGS-4.1 & Latitude & 52.134 & TAS [4] \\
\hline MIGS-4.2 & Longitude & 0.206 & TAS [4] \\
\hline MIGS-4.3 & Depth & not reported & \\
\hline MIGS-4.4 & Altitude & not reported & \\
\hline
\end{tabular}

Evidence codes - TAS: Traceable Author Statement (i.e., a direct report exists in the literature); NAS: Non-traceable Author Statement (i.e., not directly observed for the living, isolated sample, but based on a generally accepted property for the species, or anecdotal evidence). Evidence codes are from the Gene Ontology project [25]. 


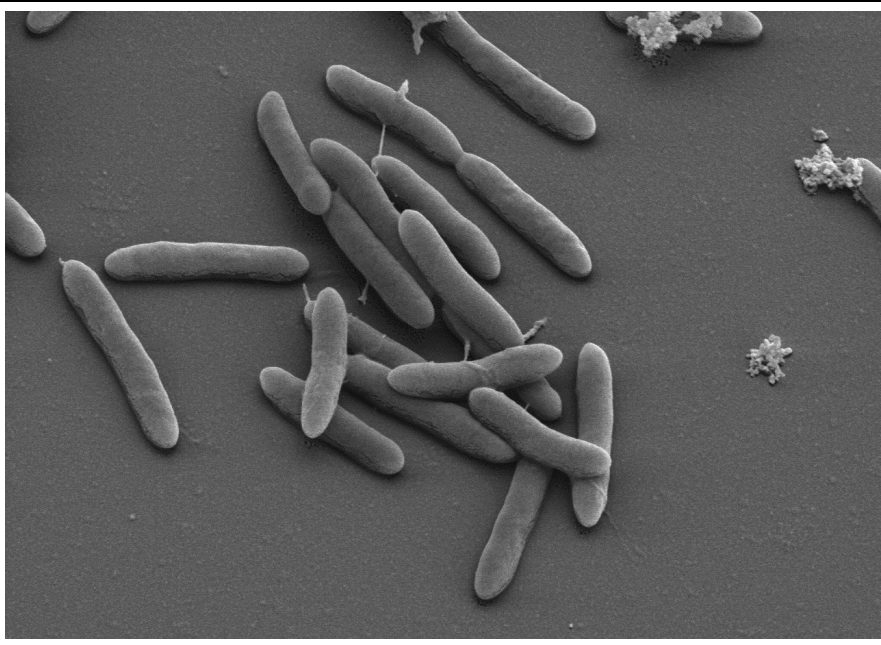

Figure 2. Scanning electron micrograph of $D$. ruminis $\mathrm{DL}^{\top}$.

Table 2. Genome sequencing project information

\begin{tabular}{lll}
\hline MIGS ID & Property & Term \\
\hline MIGS-31 & Finishing quality & Finished \\
MIGS-28 & Libraries used & Three genomic libraries: two 454 pyrosequence standard library, one \\
& & 454 PE library (9 kb insert size), one Illumina library \\
MIGS-29 & Sequencing platforms & Illumina GAii, 454 GS FLX Titanium \\
MIGS-31.2 & Sequencing coverage & $193.0 \times$ Illumina; 28.0 × pyrosequence \\
MIGS-30 & Assemblers & Newbler version 2.3, Velvet 0.7.63, phrap version SPS - 4.24 \\
MIGS-32 & Gene calling method & Prodigal 1.4, GenePRIMP \\
& INSDC ID & CP002780 \\
& Genbank Date of Release & October 12, 2011 \\
& GOLD ID & Gc01775 \\
& NCBI project ID & 47605 \\
& Database: IMG & 650716033 \\
MIGS-13 & Source material identifier & DSM 2154 \\
& Project relevance & Biotechnology, carbon cycle; sulfur cycle, metal precipitation \\
\hline
\end{tabular}

\section{Genome sequencing and assembly}

The genome was sequenced using a combination of Illumina and 454 sequencing platforms. All general aspects of library construction and sequencing can be found at the JGI website [32]. Pyrosequencing reads were assembled using the Newbler assembler (Roche). The initial Newbler assembly consisting of 74 contigs in one scaffold was converted into a phrap [33] assembly by making fake reads from the consensus, to collect the read pairs in the 454 paired end library. Illumina GAii sequencing data $(1,651.9 \mathrm{Mb})$ was assembled with Velvet [34] and the consensus sequences were shredded into $1.5 \mathrm{~kb}$ overlapped fake reads and assembled together with the 454 data. The 454 draft assembly was based on $117.7 \mathrm{Mb} 454$ draft data and all of the 454 paired end data. Newbler parameters are -consed -a 50 -l 350 -g -m -ml 20. The Phred/Phrap/Consed software package [33] was used for sequence assembly and quality assessment in the subsequent finishing process. After the shotgun stage, reads were assembled with parallel Phrap (High Performance Software, LLC). Possible mis-assemblies were corrected with gapResolution [32], Dupfinisher [35], 
or sequencing cloned bridging PCR fragments with subcloning. Gaps between contigs were closed by editing in Consed, by PCR and by Bubble PCR primer walks (J.-F. Chang, unpublished). A total of 255 additional reactions were necessary to close gaps and to raise the quality of the finished sequence. Illumina reads were also used to correct potential base errors and increase consensus quality using a software Polisher developed at JGI [36]. The error rate of the completed genome sequence is less than 1 in 100,000 . Together, the combination of the Illumina and 454 sequencing platforms provided 221-fold coverage of the genome. The final assembly contained 229,368 pyrosequence and 20,934,522 Illumina reads.

\section{Genome annotation}

Genes were identified using Prodigal [37] as part of the Oak Ridge National Laboratory genome annotation pipeline, followed by a round of manual curation using the JGI GenePRIMP pipeline [38].
The predicted CDSs were translated and used to search the National Center for Biotechnology Information (NCBI) nonredundant database, UniProt, TIGR-Fam, Pfam, PRIAM, KEGG, COG, and InterPro databases. Additional gene prediction analysis and functional annotation was performed within the Integrated Microbial Genomes - Expert Review (IMG-ER) platform [39].

\section{Genome properties}

The genome consists of one circular chromosome of 3,969,014 bp with a G+C content of $47.2 \%$ (Table 3 and Figure 3). Of the 3,986 genes predicted, 3,901 are protein-coding genes, and 85 are RNAs; 105 pseudogenes were also identified. The majority of the protein-coding genes $(67.3 \%)$ were assigned with a putative function while the remaining ones were annotated as hypothetical proteins. The distribution of genes into COGs functional categories is presented in Table 4.

Table 3. Genome Statistics

\begin{tabular}{lrr}
\hline Attribute & Value & \% of Total ${ }^{\mathbf{a}}$ \\
\hline Genome size (bp) & $3,969,014$ & $100.00 \%$ \\
DNA coding region (bp) & $3,356,856$ & $84.58 \%$ \\
DNA G+C content (bp) & $1,875,083$ & $47.24 \%$ \\
Number of replicons & 1 & \\
Extrachromosomal elements & 0 & \\
Total genes & 3,986 & $100.00 \%$ \\
RNA genes & 85 & $2.13 \%$ \\
rRNA operons & 5 & \\
Protein-coding genes & 3,901 & $97.87 \%$ \\
Pseudo genes & 105 & $2.63 \%$ \\
Genes with function prediction & 2,682 & $67.29 \%$ \\
Genes in paralog clusters & 2,015 & $50.55 \%$ \\
Genes assigned to COGs & 2,897 & $72.68 \%$ \\
Genes assigned Pfam domains & 3,047 & $76.44 \%$ \\
Genes with signal peptides & 1,077 & $27.02 \%$ \\
Genes with transmembrane helices & 1,016 & $25.49 \%$ \\
CRISPR repeats & 11 & \\
\hline
\end{tabular}




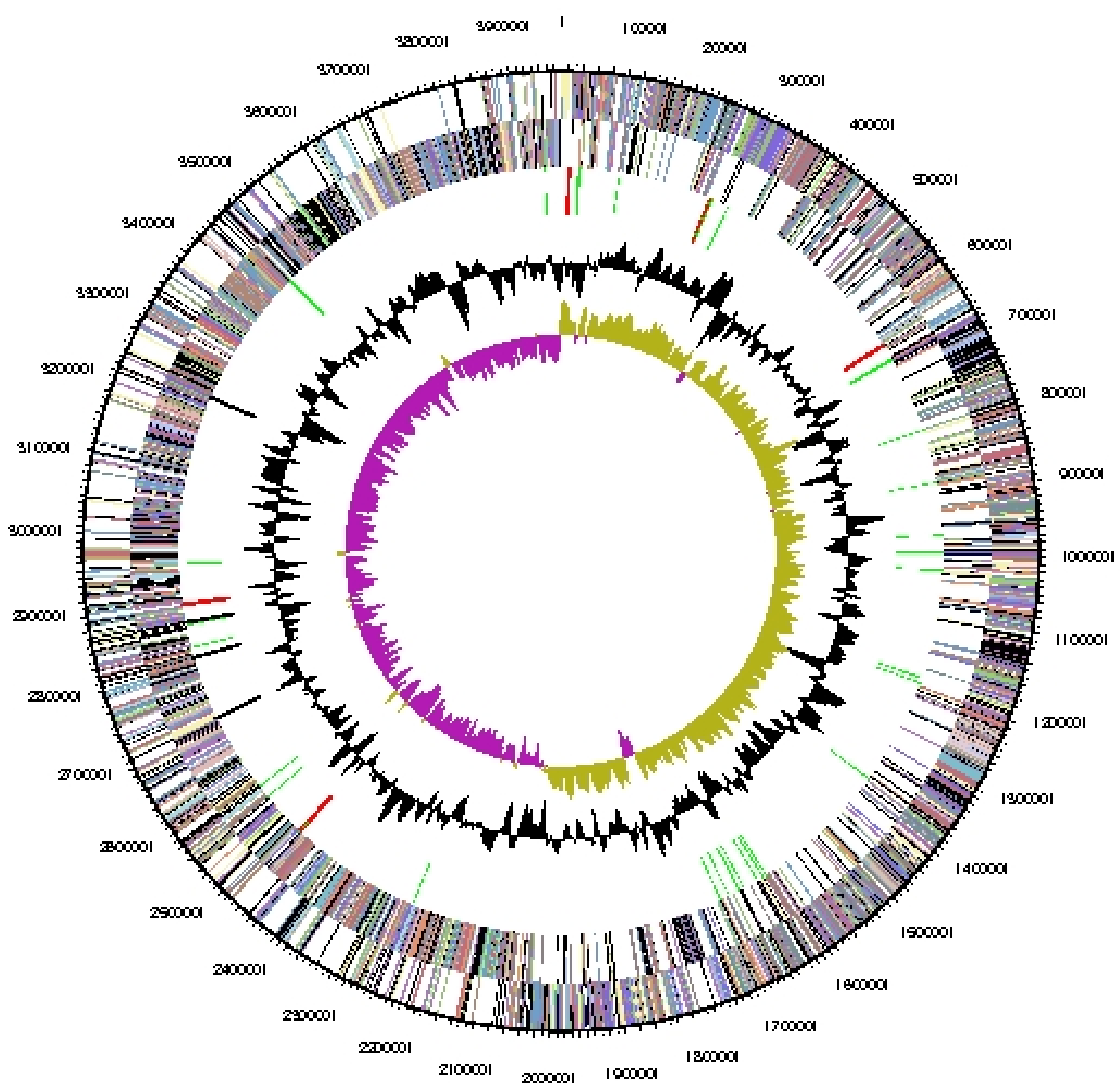

Figure 3. Graphical map of the chromosome. From outside to the center: Genes on forward strand (colored by COG categories), Genes on reverse strand (colored by COG categories), RNA genes (tRNAs green, rRNAs red, other RNAs black), GC content (black), GC skew (purple/olive).

\section{Insights into the genome sequence}

\section{Electron donor utilization}

\section{Chemoheterotrophic growth}

D. ruminis is an incomplete sulfate reducer and can metabolize various substrates as carbon and energy sources, including organic acids, alcohols and amino acids, to acetate $[1,6,40]$. In the $D$. ruminis genome, numerous genes are present that encode aminotransferases belonging to class I and II (Desru_0552, Desru_1291, Desru_1826, Desru_1950, Desru_2322, Desru_2323, Desru_3729), class III (Desru_0350, Desru_0589, Desru_3742), class IV
(Desru_1652), and class V (Desru_0021), which indicates that besides alanine, other amino acids might be substrates for this species. The oxidative deamination step of the amino acid degradation is probably catalyzed by an alanine dehydrogenase, which exists in two copies (Desru_0588 and Desru_2884) or a glutamate dehydrogenase (Desru_0556), confirming previous physiological studies [40]. 
Table 4. Number of genes associated with the general COG functional categories

\begin{tabular}{crrl}
\hline Code & Value & \%age & Description \\
\hline J & 160 & 5.0 & Translation, ribosomal structure and biogenesis \\
A & 0 & 0.0 & RNA processing and modification \\
K & 293 & 9.1 & Transcription \\
L & 177 & 5.5 & Replication, recombination and repair \\
B & 1 & 0.0 & Chromatin structure and dynamics \\
D & 49 & 1.5 & Cell cycle control, cell division, chromosome partitioning \\
Y & 0 & 0.0 & Nuclear structure \\
V & 59 & 1.8 & Defense mechanisms \\
T & 246 & 7.7 & Signal transduction mechanisms \\
M & 161 & 5.0 & Cell wall/membrane/envelope biogenesis \\
N & 88 & 2.7 & Cell motility \\
Z & 0 & 0.0 & Cytoskeleton \\
W & 0 & 0.0 & Extracellular structures \\
U & 81 & 2.5 & Intracellular trafficking, secretion, and vesicular transport \\
O & 107 & 3.3 & Posttranslational modification, protein turnover, chaperones \\
C & 252 & 7.9 & Energy production and conversion \\
G & 155 & 4.8 & Carbohydrate transport and metabolism \\
E & 279 & 8.7 & Amino acid transport and metabolism \\
F & 65 & 2.0 & Nucleotide transport and metabolism \\
H & 147 & 4.6 & Coenzyme transport and metabolism \\
I & 54 & 1.7 & Lipid transport and metabolism \\
P & 154 & 4.8 & Inorganic ion transport and metabolism \\
Q & 36 & 1.1 & Secondary metabolites biosynthesis, transport and catabolism \\
R & 351 & 10.9 & General function prediction only \\
S & 297 & 9.3 & Function unknown \\
- & 1,089 & 27.3 & Not in COGs \\
\hline
\end{tabular}

Interestingly, a taurine degradation pathway was also detected in the annotated genome. In habitats that are depleted of sulfate, like rumen or freshwater sediments, the amino sulfonic acid taurine could represent a key substrate for $D$. ruminis. Taurine is widely distributed in animal tissue, especially the large intestine, and can be converted by a taurinepyruvate aminotransferase (Desru_0589) to sulfoacetaldehyde, which in turn is cleaved by the enzyme sulfoacetaldehyde acetyltransferase (Desru_0590) into sulfite and acetyl-phosphate.
Sulfite can then be used as electron acceptor and reduced to sulfide.

Several genes encoding dehydrogenases were detected that catalyze the oxidation of organic acids (e.g., lactate), or alcohols (e.g., ethanol). The main metabolic intermediate resulting from the oxidation of organic carbon compounds in incomplete oxidizing sulfate-reducing bacteria is pyruvate, which in $D$. ruminis can be degraded by the action of several enzymes: pyruvate dehydrogenase (Desru_0066 - 0067), pyruvate-ferredoxin oxidoreductase (Desru_0099 - 0102) and pyruvate- 
formate lyase (Desru_2143 and Desru_2090). The former two enzymes are decarboxylating and yield acetyl-CoA, $\mathrm{CO}_{2}$ and reducing equivalents, and the pyruvate-formate lyase produces acetyl-CoA and formate. The latter enzyme is preferentially used during fermentative metabolism, when pyruvate is the main carbon and energy source.

Acetyl-CoA is either used for biosynthetic reactions or can be transferred into acetyl-phosphate by a phosphate acetyltransferase. However, in the annotated genome of $D$. ruminis only a phosphate butyryltransferase (Desru_2256) was found. It could be that such enzymes are not specific for butanoyl-CoA and also use acetyl-CoA. An alternative pathway for the recycling of CoA could be catalyzed by the acetyl-coenzyme A synthetase (Desru_0761). This enzyme may use AMP and pyrophosphate that are formed in the ATP-sulfurylase and APS reductase reaction, respectively, for the production of acetate, CoA and ATP, though it is not clear if this acetyl-CoA synthetase is reversible. It may also be involved in the activation of acetate during mixotrophic growth. Acetyl-phosphate, which is also produced in the degradation of taurine, is converted to ATP and acetate by the enzyme acetate kinase (Desru_1705).

Three genes involved in the acetyl-CoA pathway were not detected. These are the acetyl-CoA synthase gene $(a c s B)$, and the genes for the large and small subunit of the corrinoid iron sulfur protein. Due to the absence of these genes, $D$. ruminis is unable to perform complete oxidation of organic compounds via the acetyl-CoA pathway, which is consistent with the published species description [41].

\section{Mixotrophic growth}

Based on genes identified within the genome sequence, data hydrogen, formate and carbon monoxide could be potential substrates for mixotrophic growth in $D$. ruminis. As observed for other clostridial sulfate reducers [11] the genome of $D$. ruminis encodes several copies of [FeFe] hydrogenases, including three copies of a trimeric NAD(P)-dependent hydrogenase (Desru_2398 2396, Desru_2393 - 2391, Desru_0516 - 0514), and two copies of a membrane-associated hydrogenase (Desru_3431-3433 and Desru_0447 - 0445) that includes a TAT signal peptide that is not predicted to be cleaved off using SignalP [42], but to form a transmembrane helix that anchors the protein to the extracytoplasmic side of the membrane.
A monomeric [FeFe] hydrogenase (Desru_2180) and a hydrogenase encoding a PAS-sensing domain (Desru_2509), similar to HsfB [43] are also present. In addition, the utilization of hydrogen may also be catalyzed by a $\mathrm{Ni}, \mathrm{Fe}$ hydrogenase encoded by the genes Desru_2370 - 2372. This enzyme is bound to the membrane by a cytochrome $b$, but seems to be cytoplasmic as no signal peptides are predicted. Two gene loci encoding formate dehydrogenases are located adjacently in the genome. Genes Desru_3012 - 3008 code for a membraneassociated enzyme in which the catalytic subunit is coded by three genes (Desru_3012 - 3010), as observed in other organisms. The first gene (Desru_3012) includes a TAT signal peptide, so the localization of the enzyme relative to the membrane will depend on whether this peptide associates with the catalytic subunit (Desru_3010) or not. The gene Desru_3011 encodes for the FeS domain of the catalytic subunit. The second formate dehydrogenase (Desru_3002-3005) is a tetrameric NAD(P)-dependent enzyme. Potential genes encoding the catalytic subunit of anaerobic-type carbon monoxide dehydrogenases (coos) were identified at Desru_0859 and Desru_3320. However, no other CODH complex genes were found near either of the two $\operatorname{coo} S$ genes, except for cooC at Desru_0860.

While growth with hydrogen and formate with acetate as carbon source was confirmed in laboratory experiments, no growth was obtained with 5\% $(\mathrm{v} / \mathrm{v})$ of carbon monoxide in the headspace gas atmosphere [23]. This is in contrast with the cooS present in the genome and brings into question the function of this gene in $D$. ruminis. In a study about the fermentation burst in Desulfovibrio vulgaris Hildenborough it was found that $\mathrm{CO}$ is produced at low levels during growth on pyruvate or lactate [41]. It was hypothesized that the catalytic subunit of carbon monoxide dehydrogenase could be involved in an internal metabolism or cycling of carbon monoxide. In $D$. vulgaris, the $\operatorname{coo} S$ gene (YP_011311.1) is downstream of a transcriptional regulator (YP_011310.1) and upstream of the cooC gene (YP_011312.1). This localization is similar to what we find in D. ruminis, Desru_0859, Desru_0858 and Desru_0860, respectively. Thus, carbon-monoxide dehydrogenases could play a role in the internal metabolism or cycling of carbon monoxide during growth of $D$. ruminis on organic acids. However, in contrast to D. vulgaris, no COinduced hydrogenase ( $\mathrm{coo}$ ) is present in D. ruminis. 


\section{Energy metabolism}

The genome of $D$. ruminis encodes the full set of genes necessary for dissimilatory sulfate reduction as well as several membrane complexes, which deliver electrons from membrane electron carriers like menaquinol to cytosolic sulfatereducing enzymes. The following genes encoding cytoplasmic enzymes for dissimilatory sulfate reduction were detected in the $D$. ruminis genome: Sulfate adenylyltransferase (ATP-sulfurylase, Desru_3378), adenosine-5'-phosphosulfate (APS) reductase (Desru_3376 - 3377) and dissimilatory sulfite reductase (Desru_0386 - 0387 and Desru_3723 - 3724). In D. ruminis, like in most sulfate reducers, ATP-sulfurylase and APS reductase are present as one copy. However, in contrast to most other sulfate reducers, $D$. ruminis contains two copies of the $d s r A B D$ genes. As observed in other Desulfotomaculum species, the alpha subunit of the APS reductase appears to be membraneanchored.

The generation of a proton gradient across the cytoplasmic membrane is thought to be the main mechanism for generation of energy in sulfatereducing bacteria. The coupling of the reduction of sulfate to sulfide, which occurs exclusively in the cytoplasm with a membrane-bound electron transport chain, and a vectorial proton transport across the membrane is still far from being understood. Electrons and protons required for the generation of a chemiosmotic gradient in Grampositive sulfate reducers could be generated by the oxidation of small intermediate metabolites, like hydrogen, $\mathrm{CO}^{-}$or formate at the cytoplasmic membrane. Several membrane-bound enzyme complexes were recently identified that could play a role in this process.

A membrane-bound pyrophosphatase (Desru_3593) could use the energy generated from the cleavage of pyrophosphate, which is formed in the activation of sulfate by the ATPsulfurylase, for proton translocation.

The QmoAB complex (Desru_3374 - 3375) is suggested to play a role in donating electrons to the APS reductase [44], and the genes of both enzymes are located next to each other in the $D$. ruminis genome. The gene for the membrane subunit QmoC is absent, as in other clostridial sulfate reducers. The QmoA subunit is predicted to contain a signal peptide, but this likely forms a transmembrane helix, as it is still present in the mature protein [45], which indicates a localization at the inner surface of the cytoplasmic membrane. In Gram-negative sulfate-reducing bacteria, a transmembrane DsrMKJOP complex is conserved, which probably transfers electrons from the periplasmic space to the dissimilatory sulfite reductase. In Gram-positive sulfate reducers, only a truncated DsrMK complex seems to be present [46], which is encoded adjacent to a small soluble protein designated DsrC that is proposed to have a function in shuttling electrons from DsrK to the cytoplasmic DsrAB sulfite reductase [47]. In $D$. ruminis this enzyme system is encoded by the genes Desru_3734 - 3736, and a second copy of the $d s r M K$ genes is present (Desru_2596 - 2597)

The two $c$-type cytochromes present in " $D$. reducens" and annotated as a nitrite reductase are absent in D. ruminis, which is consistent with the other Desulfotomaculum species sequenced to date (except D. nigrificans) [46].

An energy-conserving NADH-quinone oxidoreductase (Complex I, Desru_1808 - 1818 and Desru_0514 - 516) is present, which will couple NADH oxidation to proton translocation. Furthermore, a multimeric membrane-bound complex was identified at Desru_3260 - 3265, that belongs to the family of Ehr complexes (for energyconserving hydrogenase related complex) first identified in Geobacter spp., but present in many microorganisms [48,49]. The subunits of Ehr complexes are related to subunits of complex I and the Ech energy conserving hydrogenases, but in most cases the cysteines binding the NiFe cluster are absent, so these complexes are not real hydrogenases. In D. ruminis EhrL (Desru_3264) the four Cys required to coordinate the catalytic center are present, so this complex may be a true energy-conserving hydrogenase. The proton gradient resulting from the above-mentioned reactions is used by a $\mathrm{F}_{0} \mathrm{~F}_{1}$-type ATP synthase complex encoded by the genes Desru_3687 - 3694.

There are number of heterodisulfide reductases in the genome: three loci were identified which contained hdrA (Desru_0205, Desru_0212 and Desru_3375) and $h d r B$ (Desru_3379 and Desru_2699) and hdrC-like (Desru_3380 and Desru_2700) heterodisulfide reductases. In addition, a fused $h d r A$ with $m v h \mathrm{D}$ (methyl-viologen reducing hydrogenase delta subunit) was identified (Desru_3374). 


\section{Comparative genomics}

We analyzed the fraction of shared genes in three genomes of Desulfotomaculum species with validly published names. The genomes of $D$. acetoxidans [50] and D. ruminis [10] are complete, whereas the genome of the type species $D$. nigrificans is only available as a draft sequence. D. nigrificans has the smallest genome with 3,014 protein coding sequences. The resulting data are illustrated in the Venn diagram shown in [Figure 4]. The largest overlap is found between the strains $D$. nigrificans DSM $574^{\mathrm{T}}$ and D. ruminis DSM $2154^{\mathrm{T}}$, which share 2,359 homologous proteins corresponding to $78.3 \%$ of the DSM $574^{\mathrm{T}}$ genes and $60.5 \%$ of the DSM $2154^{\mathrm{T}}$ genes. Thus, a closer relationship between D. ruminis DSM $2154^{\mathrm{T}}$ and D. nigrificans DSM $574^{\mathrm{T}}$ than between D. acetoxidans DSM $771^{\mathrm{T}}$ and D. nigrificans DSM $574^{\mathrm{T}}$, as suggested by the $16 \mathrm{~S}$ rRNA based phylogenetic tree, is confirmed by whole-genome data.
Figures $5 \mathrm{~A}$ and $5 \mathrm{~B}$ show the organization of $d s r \mathrm{AB}$ (A), qmoBA, aprAB and $h d r \mathrm{BC}(\mathrm{B})$ and neighboring genes for $D$. ruminis, " $D$. reducens" and $D$. acetoxidans. In Figure $5 d s r \mathrm{D}$ is upstream of $d s r \mathrm{AB}$ in all three strains. However, no other neighboring genes are similar to each other. In contrast, Figure 5B shows remarkable homology in gene organization for the aprAB gene neighborhood for $D$. ruminis and "D. reducens". Gene sequence is also very similar for that region (53-94\% identity for the genes displayed including hypothetical proteins) which suggests horizontal gene transfer from a common ancestor. The $d s r \mathrm{AB}$ and aprBA proteins of $D$. ruminis are more closely related to "D. reducens" than to D. acetoxidans [Figure 6A and 6B]. This is in accordance with the 16SrRNA based phylogenetic tree and the whole-genome data.

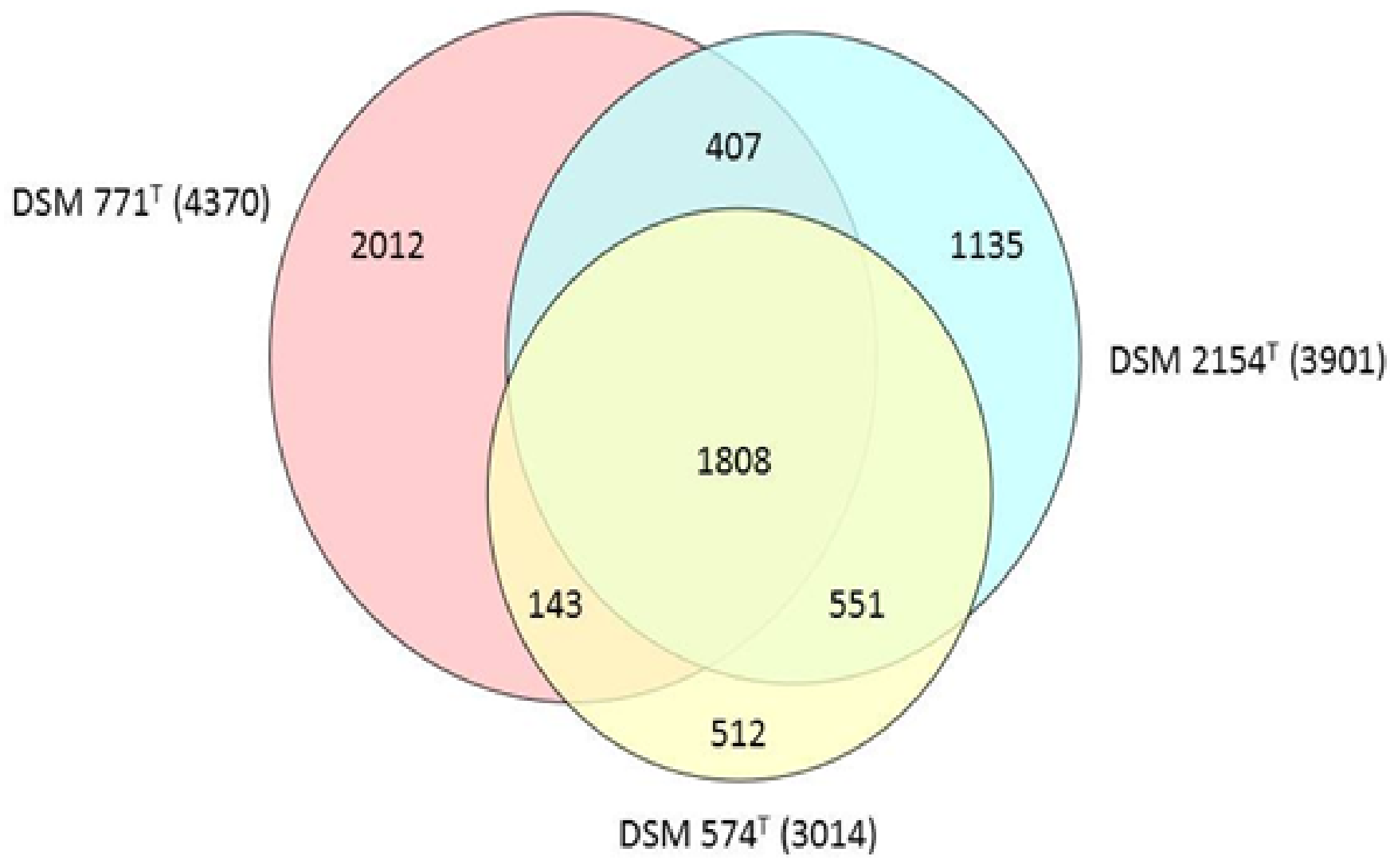

Figure 4. Venn diagram showing a comparison of three different Desulfotomaculum genomes, D. ruminis DSM $2154^{\top}, D$. acetoxidans DSM $771^{\top}$ and D. nigrificans DSM $564^{\top}$. The number of overlapping protein genes is given inside the areas of the circles and the total number of derived protein sequences used for each strain is shown in parentheses. The figure was created using the program Venn diagram plotter available from the Pacific Northwest National Laboratory Software Distribution Center [51]. 


\section{D. ruminis}

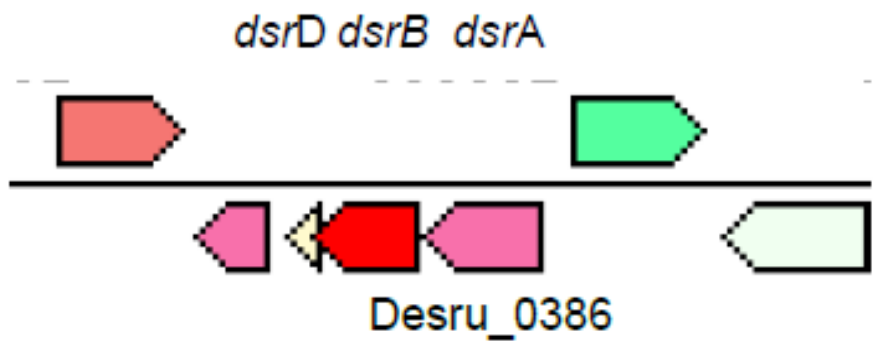

D. reducens

$d s r D$ dsrB dsrA

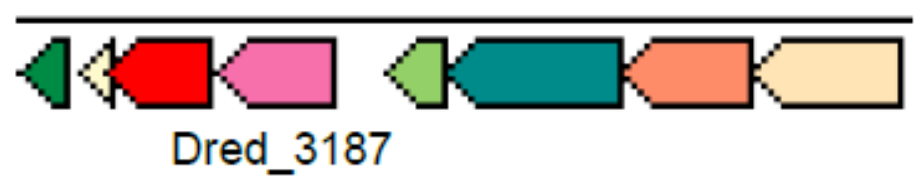

D. acetoxidans

$d s r D d s r B$ dsrA

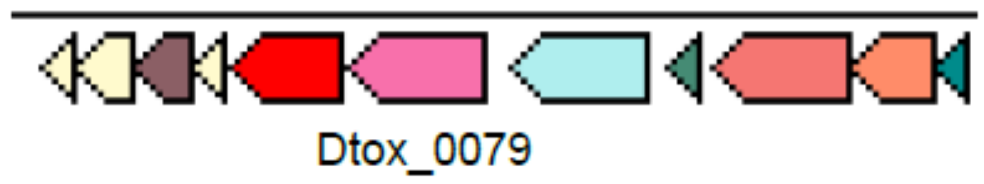

Figure 5A. Organization of $d s r \mathrm{AB}$ and neighboring genes for three Desulfotomaculum species. Other genes are indicated by their locus tags.

D. ruminis

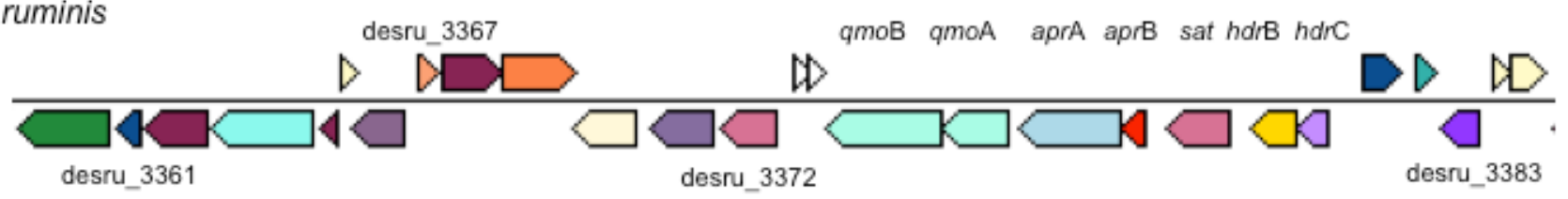

D. reducens

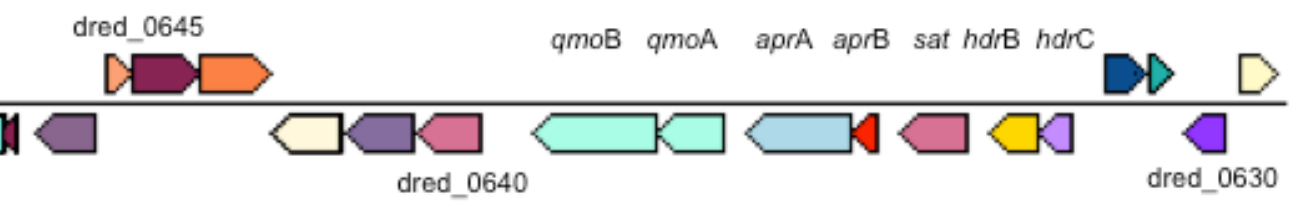

D. acetoxidans

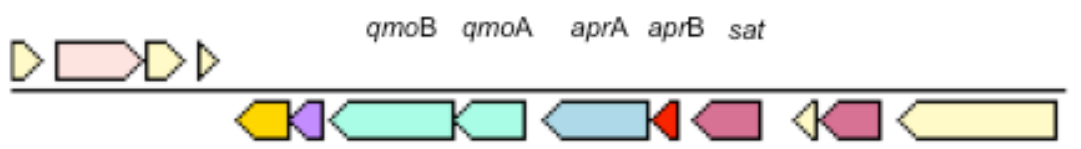

Figure 5B. Organization of qmoBA, aprAB and hdrBC and neighboring genes for three Desulfotomaculum species. Other genes are indicated by their locus tags. 


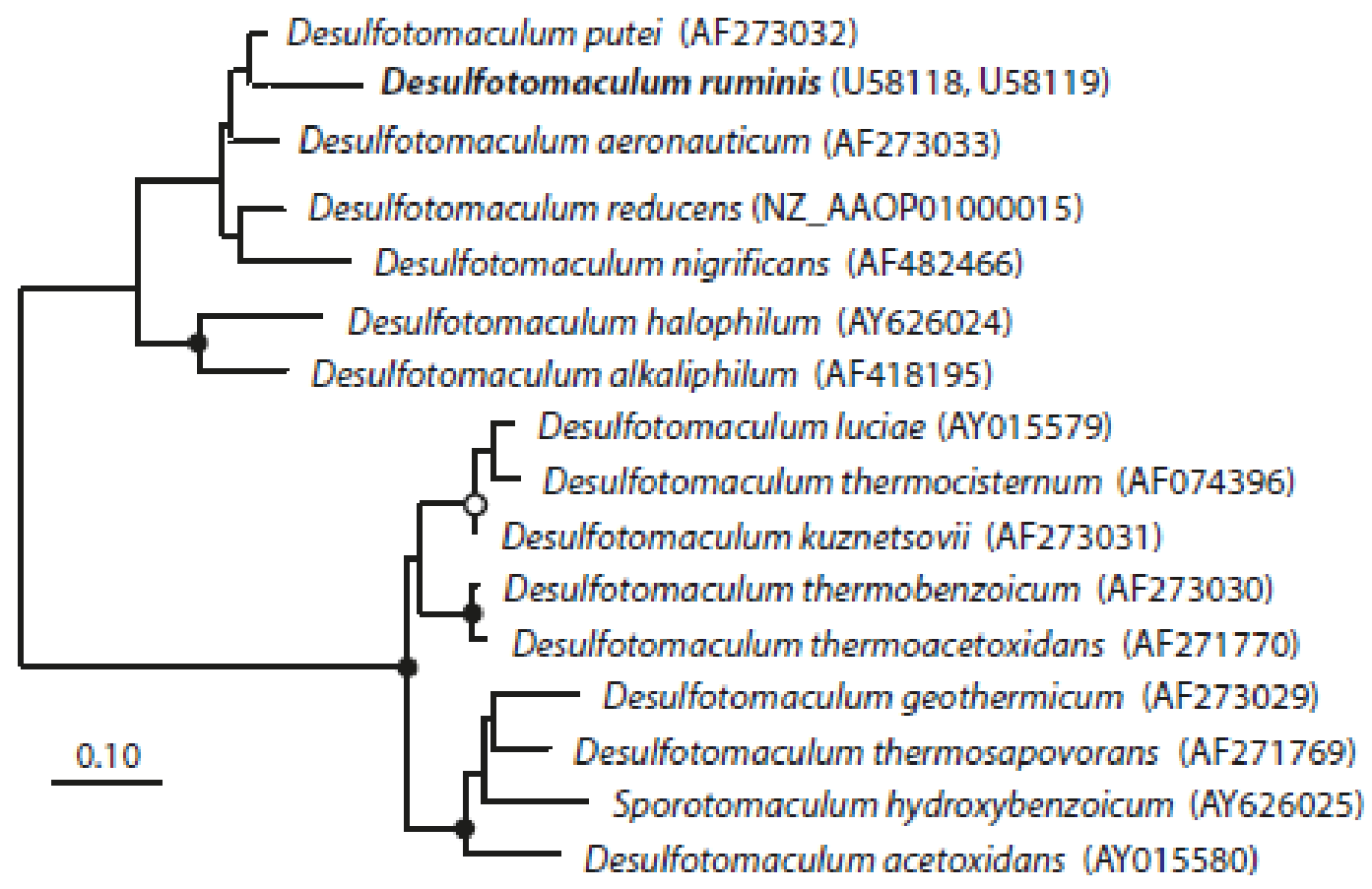

Figure 6A. Phylogenetic tree of the dsrAB protein sequences. The trees $(6 \mathrm{~A}$ and $6 \mathrm{~B})$ were inferred from proteins sequences using RAxML (maximum-likelihood) in the software program ARB. The sequences of Archaeoglobus fulgidus, A. profundus, and A. veneficus were used as outgroup, but were pruned from the tree. The sequence of $D$. ruminis is written in bold. The black circles are bootstrap values between $100-75 \%$, the white circles are values between $75-50 \%$. The scale bar corresponds to $10 \%$ estimated sequence divergence.

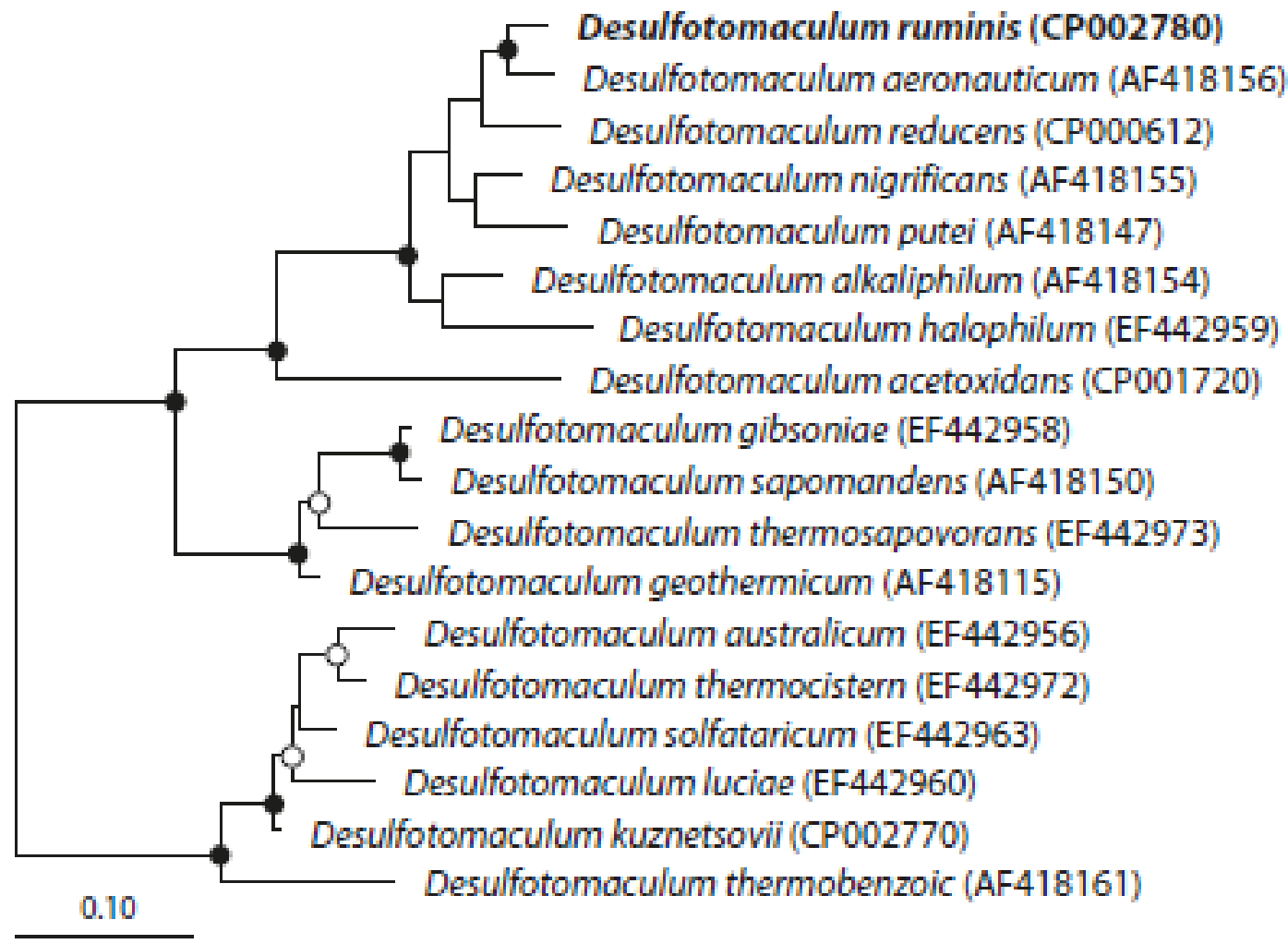

Figure 6B. Phylogenetic tree of the aprBA protein sequence. 


\section{Acknowledgements}

We would like to gratefully acknowledge the help of Maren Schröder for growing D. ruminis cultures and Susanne Schneider for DNA extraction (both at DSMZ). The work conducted by the U.S. Department of Energy Joint Genome Institute was supported by the Office of

\section{References}

1. Campbell LL, Postgate JR. Classification of the spore-forming sulfate-reducing bacteria. Bacteriol Rev 1965; 29:359-363. PubMed

2. Euzéby JP. List of bacterial names with standing in nomenclature: A folder available on the Internet. Int J Syst Bacteriol 1997; 47:590-592. PubMed http://dx.doi.org/10.1099/00207713-47-2-590

3. Garrity G. NamesforLife. BrowserTool takes expertise out of the database and puts it right in the browser. Microbiol Today 2010; 37:9.

4. Coleman GS. A sulphate-reducing bacterium from the sheep rumen. J Gen Microbiol 1960; 22:423436. PubMed http://dx.doi.org/10.1099/00221287-22-2-423

5. Lewis D. The reduction of sulphate in the rumen of the sheep. Biochem J 1954; 56:391-399. PubMed

6. Campbell LL, Singleton R, Jr. Genus IV. Desulfotomaculum Campbell and Postgate 1965, 361 AL. In: Bergey's Manual of Systematic Bacteriology, vol. 2. 1st ed. Edited by: Holt JG. The Williams and Wilkins Co., Baltimore; 1974; pp 572-573.

7. Altschul SF, Gish W, Miller W, Myers E, Lipman D. Basic local alignment search tool. J Mol Biol 1990; 215:403-410. PubMed

8. Korf I, Yandell M, Bedell J. BLAST, O'Reilly, Sebastopol, 2003

9. DeSantis TZ, Hugenholtz P, Larsen N, Rojas M, Brodie EL, Keller K, Huber T, Dalevi D, Hu P, Andersen GL. Greengenes, a chimera-checked $16 \mathrm{~S}$ rRNA gene database and workbench compatible with ARB. Appl Environ Microbiol 2006; 72:5069-5072. PubMed http://dx.doi.org/10.1128/AEM.03006-05

10. Pereira IA, Ramos AR, Grein F, Marques MC, da Silva SM, Venceslau SS. A comparative genomic analysis of energy metabolism in sulfate reducing bacteria and archaea. Front Microbiol 2011; 2:69. PubMed

11. Savoy de Giori G, Font de Valdéz G, Pesce de Ruiz Holgado A, Oliver G. 1982 Isolation and identification of anaerobic contaminants from a
Science of the U.S. Department of Energy under Contract No. DE-AC02-05CH11231, and was also supported by grants CW-TOP 700.55.343 and ALW 819.02.014 of the Netherlands Science Foundation (NWO).

machine for producing processed cheese. Rev Argent Microbiol 1982; 14:105-110. PubMed

12. Chang YJ, Peacock AD, Long PE, Stephen JR, McKinley JP, MacNaughton SJ. Anwar Hussain AKM, Saxton AM, White DC. Diversity and characterization of sulfate-reducing bacteria in groundwater at a uranium mill tailings site. Appl Environ Microbiol 2001; 67:3149-3160. PubMed http://dx.doi.org/10.1128/AEM.67.7.3149$\underline{3160.2001}$

13. Field D, Garrity G, Gray T, Morrison N, Selengut J, Sterk P, Tatusova T, Thomson N, Allen MJ, Angiuoli SV, et al. The minimum information about a genome sequence (MIGS) specification. Nat Biotechnol 2008; 26:541-547. PubMed http://dx.doi.org/10.1038/nbt1360

14. Woese CR, Kandler O, Wheelis ML. Towards a natural system of organisms: proposal for the domains Archaea, Bacteria, and Eucarya. Proc Natl Acad Sci USA 1990; 87:4576-4579. PubMed http://dx.doi.org/10.1073/pnas.87.12.4576

15. Gibbons NE, Murray RGE. Proposals Concerning the Higher Taxa of Bacteria. Int I Syst Bacteriol 1978; 28:1-6.

http://dx.doi.org/10.1099/00207713-28-1-1

16. Garrity GM, Holt JG. The Road Map to the Manual. In: Garrity GM, Boone DR, Castenholz RW (eds), Bergey's Manual of Systematic Bacteriology, Second Edition, Volume 1, Springer, New York, 2001, p. 119-169.

17. Murray RGE. The Higher Taxa, or, a Place for Everything...? In: Holt JG (ed), Bergey's Manual of Systematic Bacteriology, First Edition, Volume 1, The Williams and Wilkins Co., Baltimore, 1984, p. 31-34.

18. List of new names and new combinations previously effectively, but not validly, published. List no. 132. Int I Syst Evol Microbiol 2010; 60:469472. http://dx.doi.org/10.1099/ijs.0.022855-0

19. Rainey FA. Class II. Clostridia class nov. In: De Vos P, Garrity G, Jones D, Krieg NR, Ludwig W, Rainey FA, Schleifer KH, Whitman WB (eds), Bergey's Manual of Systematic Bacteriology, Se- 
cond Edition, Volume 3, Springer-Verlag, New York, 2009, p. 736.

20. Skerman VBD, McGowan V, Sneath PHA. Approved Lists of Bacterial Names. Int J Syst Bacteriol 1980; 30:225-420. http://dx.doi.org/10.1099/00207713-30-1-225

21. Prévot AR. In: Hauderoy P, Ehringer G, Guillot G, Magrou. J., Prévot AR, Rosset D, Urbain A (eds), Dictionnaire des Bactéries Pathogènes, Second Edition, Masson et Cie, Paris, 1953, p. 1-692.

22. Rogosa M. Peptococcaceae, a new family to include the Gram-positive, anaerobic cocci of the genera Peptococcus, Peptostreptococcus and Ruminococcus. Int J Syst Bacteriol 1971; 21:234237. http://dx.doi.org/10.1099/00207713-21-3$\underline{234}$

23. Klemps R, Cypionka H, Widdel F, Pfennig N. Growth with hydrogen, and further physiological characteristics of Desulfotomaculum species. Arch Microbiol 1985; 143:203-208. http://dx.doi.org/10.1007/BF00411048

24. BAuA. Classification of bacteria and archaea in risk groups. TRBA 466. p. 74. Bundesanstalt für Arbeitsschutz und Arbeitsmedizin, Germany. 2010

25. Ashburner M, Ball CA, Blake JA, Botstein D, Butler $\mathrm{H}$, Cherry JM, Davis AP, Dolinski K, Dwight SS, Eppig JT, et al. Gene Ontology: tool for the unification of biology. Nat Genet 2000; 25:25-29. PubMed http://dx.doi.org/10.1038/75556

26. Hagenauer A, Hippe H, Rainey FA. Desulfotomaculum aeronauticum sp. nov., a sporeforming, thiosulfate-reducing bacterium from corroded aluminium alloy in an aircraft. Syst Appl Microbiol 1997; 20:65-71. http://dx.doi.org/10.1016/S0723-2020(97)80049$\underline{5}$

27. Collins MD, Widdel F. Respiratory quinones of sulphate-reducing and sulphur-reducing bacteria: a systematic investigation. Syst Appl Microbiol 1986; 8:8-18. http://dx.doi.org/10.1016/S0723$\underline{\text { 2020(86)80141-2 }}$

28. Pagani I, Liolios K, Jansson J, Chen IM, Smirnova T, Nosrat B, Markowitz VM, Kyrpides NC. The Genomes OnLine Database (GOLD) v.4: status of genomic and metagenomic projects and their associated metadata. Nucleic Acids Res 2012;

40:D571-D579. PubMed

http://dx.doi.org/10.1093/nar/gkr1100

29. List of growth media used at DSMZ: http://www.dsmz.de/catalogues/catalogue- microorganisms/culture-technology/list-of-mediafor-microorganisms.html

30. Wu D, Hugenholtz P, Mavromatis K, Pukall R, Dalin E, Ivanova NN, Kunin V, Goodwin L, Wu $M$, Tindall BJ, et al. A phylogeny-driven genomic encyclopaedia of Bacteria and Archaea. Nature 2009; 462:1056-1060. PubMed http://dx.doi.org/10.1038/nature08656

31. Gemeinholzer B, Dröge $G$, Zetzsche $H$, Haszprunar G, Klenk HP, Güntsch A, Berendsohn WG, Wägele JW. The DNA Bank Network: the start from a German initiative. Biopreserv Biobank $2011 ; 9: 51-55$. http://dx.doi.org/10.1089/bio.2010.0029

32. JGI website. http://www.jgi.doe.gov/

33. The Phred/Phrap/Consed software package. http://www.phrap.com

34. Zerbino DR, Birney E. Velvet: algorithms for de novo short read assembly using de Bruijn graphs. Genome Res 2008; 18:821-829. PubMed http://dx.doi.org/10.1101/gr.074492.107

35. Han C, Chain P. Finishing repeat regions automatically with Dupfinisher. In: Proceeding of the 2006 international conference on bioinformatics \& computational biology. Arabnia HR, Valafar H (eds), CSREA Press. June 26-29, 2006: 141-146.

36. Lapidus A, LaButti K, Foster B, Lowry S, Trong S, Goltsman E. POLISHER: An effective tool for using ultra short reads in microbial genome assembly and finishing. AGBT, Marco Island, FL, 2008

37. Hyatt D, Chen GL, LoCascio PF, Land ML, Larimer FW, Hauser LJ. Prodigal: prokaryotic gene recognition and translation initiation site identification. BMC Bioinformatics 2010; 11:119. PubMed http://dx.doi.org/10.1186/1471-2105-11-119

38. Pati A, Ivanova NN, Mikhailova N, Ovchinnikova G, Hooper SD, Lykidis A, Kyrpides NC.

GenePRIMP: a gene prediction improvement pipeline for prokaryotic genomes. Nat Methods 2010; 7:455-457. PubMed http://dx.doi.org/10.1038/nmeth.1457

39. Markowitz VM, Ivanova NN, Chen IMA, Chu K, Kyrpides NC. IMG ER: a system for microbial genome annotation expert review and curation. Bioinformatics 2009; 25:2271-2278. PubMed http://dx.doi.org/10.1093/bioinformatics/btp393

40. Stams AJM, Hansen TA. Metabolism of L-alanine in Desulfotomaculum ruminis and two marine Desulfovibrio strains. Arch Microbiol 1986; 145:277-279. http://dx.doi.org/10.1007/BF00443658 
41. Voordouw G. Carbon monoxide cycling by Desulfovibrio vulgaris Hildenborough. J Bacteriol 2002; 184:5903-5911. PubMed http://dx.doi.org/10.1128//B.184.21.59035911.2002

42. Petersen TN, Brunak S, von Heijne G, Nielsen H. SignalP 4.0: discriminating signal peptides from transmembrane regions. Nat Methods 2011; 8:785-786. PubMed http://dx.doi.org/10.1038/nmeth.1701

43. Shaw AJ, Hogsett DA, Lynd LR. Identification of the [FeFe]-hydrogenase responsible for hydrogen generation in Thermoanaerobacterium saccharolyticum and demonstration of increased ethanol yield via hydrogenase knockout. J Bacteriol 2009; 191:6457-6464. PubMed http://dx.doi.org/10.1128//B.00497-09

44. Ramos AR, Keller KL, Wall JD, Pereira IA. The membrane QmoABC complex interacts directly with the dissimilatory adenosine $5^{\prime}$ phosphosulfate reductase in sulfate reducing bacteria. Front Microbiol 2012; 3:137. PubMed

45. Pires RH, Lourenco Al, Morais F, Teixeira M, Xavier AV, Saraiva LM, Pereira IA. A novel membrane-bound respiratory complex from Desulfovibrio desulfuricans ATCC 27774.

Biochim Biophys Acta 2003; 1605:67-82. PubMed http://dx.doi.org/10.1016/S00052728(03)00065-3

46. Junier P, Junier T, Podell S, Sims DR, Detter JC, Lykidis A, Han CS, Wigginton NS, Gaasterland T, Bernier-Latmani R. The genome of the Grampositive metal- and sulfate-reducing bacterium Desulfotomaculum reducens strain MI-1. Environ Microbiol 2010; 12:2738-2754. PubMed
47. Oliveira TF, Vonrhein C, Matias PM, Venceslau SS, Pereira IA, Archer M. The crystal structure of Desulfovibrio vulgaris dissimilatory sulfite reductase bound to DsrC provides novel insights into the mechanism of sulfate respiration. J Biol Chem 2008; 283:34141-34149. PubMed http://dx.doi.org/10.1074/jbc.M805643200

48. Oliveira TF, Vonrhein C, Matias PM, Venceslau SS, Pereira IA, Archer M. The crystal structure of Desulfovibrio vulgaris dissimilatory sulfite reductase bound to DsrC provides novel insights into the mechanism of sulfate respiration. / Biol Chem 2008; 283:34141-34149. PubMed http://dx.doi.org/10.1074/jbc.M805643200

49. Coppi MV. The hydrogenases of Geobacter sulfurreducens: a comparative genomic perspective. Microbiology 2005; 151:1239-1254. PubMed http://dx.doi.org/10.1099/mic.0.27535-0

50. Spring S, Lapidus A, Schröder M, Gleim D, Sims D, Meinecke L, Glavina Del Rio T, Tice H, Copeland A, Cheng JF, et al. Complete genome sequence of Desulfotomaculum acetoxidans type strain $\left(5575^{\mathrm{T}}\right)$. Stand Genomic Sci 2009; 1:242253. PubMed http://dx.doi.org/10.4056/sigs.39508

51. Venn diagram plotter available from the Pacific Northwest National Laboratory Software Distribution Center: $\underline{\text { http://omics.pnl.gov }}$

52. Vignais PM, Billoud B. Occurrence, classification, and biological function of hydrogenases: an overview. Chem Rev 2007; 107:4206-4272. PubMed http://dx.doi.org/10.1021/cr050196r 